Revista de Psicología y Educación / Journal of Psychology and Education, 2022, 17(1), $44-53$ (www.rpye.es) Doi: https://doi.org/10.23923/rpye2022.01.215

ISSN: $1699-9517$

\title{
¿Qué Sabemos Sobre los Estilos Educativos Parentales y los Trastornos en la Infancia y Adolescencia? Una Revisión de la Literatura
}

\author{
Natalia Martín, Marisol Cueli, Laura M. Cañamero y Paloma González-Castro
}

Universidad de Oviedo

\begin{abstract}
Resumen: La familia es el entorno más importante donde las personas aprenden a socializarse y en la que el Estilo Educativo Parental puede presentar consecuencias en el desarrollo evolutivo del niño a diferentes niveles. El objetivo del presente trabajo fue realizar una revisión a lo largo de las aportaciones más relevantes en la literatura científica en relación con los Estilos Educativos Parentales y los trastornos en la infancia y adolescencia. Se analizó la literatura existente desde 2011 hasta 2021, mediante una búsqueda realizada en la Web of Science, Google Académico, Psyclnfo, Pubmed. Se seleccionaron 16 estudios para su revisión. Los resultados de estos trabajos mostraron una tendencia en la que los Estilos Educativos Parentales caracterizados por el afecto y la implicación parental, promueven un mejor ajuste psicosocial y correlacionan en menor medida con la aparición de problemas o trastornos de índole internalizante y externalizante.
\end{abstract}

Palabras clave: Familia; Estilos Educativos Parentales; Ajuste Emocional; Infancia; Adolescencia

\section{What Do We Know About Parental Educational Styles and Disorders in Childhood and Adolescence? A Literature Review}

Abstract: The family is the most important environment in which people learn to socialise and the parental educational style can have important consequences on the child's development at different levels. The present study aimed to carry out a review of the most relevant contributions in the scientific literature about parental educational styles and disorders in childhood and adolescence. After a search conducted in the Web of Science, Google Scholar, Psyclnfo, and Pubmed, the existing literature from 2011 to 2021 was analysed. Sixteen studies were selected for review. The results of these studies showed a trend in which parental educational styles characterised by parental affection and involvement promote better psychosocial adjustment and correlate to a lesser extent with the onset of internalising and externalising problems or disorders.

Key words: Family; Parental Educational Styles; Emotional Adjustment; Childhood; Adolescence

El término familia se encuentra en constante transformación, fundamentalmente debido a los cambios ocurridos en las últimas décadas (demográficos, laborales, culturales, económicos, nuevas tipologías familiares, y cambios de roles). Siguiendo a Rodrigo y Palacios (2014), el término familia haría referenciaa launión de personas que comparten

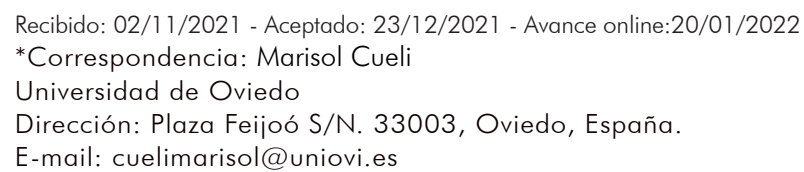

un proyecto vital caracterizado por el deseo de durabilidad, sentimientos de pertenencia, compromiso entre sus miembros, intimidad y reciprocidad. La Declaración Universal de los Derechos Humanos, hace referencia a la familia como un elemento natural, universal y fundamental de la sociedad, y que tiene derecho a la protección de la sociedad y del Estado (Asamblea General de las Naciones Unidas, 1948). Además, siguiendo a Fontana et al. (2013) en la actualidad cada vez más se trata de revalorizar la función social de 
la familia y en definitiva la socialización desde el ámbito familiar considerando sus efectos sobre el desarrollo de los niños. En el presente trabajo trataremos de responder a la cuestión, ¿̇cómo la relación entre padres e hijos se asocia con los trastornos de la infancia y la adolescencia? Para ello se realizará una revisión de la literatura después de contextualizar esta temática en torno a los conceptos de la socialización y los Estilos Educativos Parentales.

Diferentes autores definen la socialización como el proceso en el cual los individuos incorporan normas, roles, valores, actitudes y creencias, a partir del contexto sociohistórico en el que se encuentran inmersos (Grusec y Hastings, 2007). Siguiendo a Cava y Musitu (2001), tanto la infancia como la adolescencia son fundamentales en este proceso de socialización, es por ello, que la familia se sitúa en una posición de privilegio en tanto que transmisora de pautas culturales. Tradicionalmente, la socialización se concebía en una sola dirección, los padres eran los que influían sobre los hijos (Bersabé et al., 2001). Sin embargo, tal y como se va a plantear en este trabajo, desde hace ya varios años se viene considerando el proceso de socialización como una relación bidireccional, considerando que también los hijos influyen sobre sus padres a lo largo de toda la vida (Franco et al., 2014; Oliva et al., 2008).

En el concepto de socialización es preciso destacar que existen diferentes variables relevantes relacionadas con la relación de padres e hijos como el tono de la relación, el mayor o menor nivel de comunicación (aceptación-rechazo, calor-frialdad, afectohostilidad, proximidad-distanciamiento) y las conductas dirigidas a encauzar el comportamiento del niño (autonomía-control, flexibilidad-rigidez, permisividad-restricción). En definitiva, son diversas las variables de socialización fundamentales o dimensiones que combinadas se han formulado para presentar unos Estilos Educativos Parentales determinados (Torío-López et al., 2008).

\section{LOS ESTILOS EDUCATIVOS PARENTALES}

El concepto de Estilo Educativo Parental ha venido asociado a múltiples términos y expresiones como estilos de crianza, pautas de crianza, estilos parentales, estilos de socialización y modelos de crianza (IzzedinBouquet y Pachaoja-Londoño, 2009). El Estilo Educativo Parental trata de indicar unas coordenadas o ejes de regulación dentro de las que se enmarcan las estrategias educativas y de socialización que los padres ejercen sobre los hijos (Aroca-Montolío y Cánovas-Leonhardt, 2012). Cabe destacar que los Estilos Educativos Parentales están determinados por un gran número de factores, tales como el número de hijos, el género de cada uno, la ubicación en el orden (hijo mayor o menor), la salud y la apariencia física o aspectos culturales y religiosos de la propia familia (Jorge y González, 2017; Torío-López et al., 2008).

Distintos modelos teóricos han tratado de explicar los Estilos Educativos Parentales desde finales de la década de los 60 y principios de los 70 (Torío-López et al., 2008). Desde esas fechas, se ha ido modificando de manera evidente la visión de dichos modelos teóricos hasta llegar a un enfoque que hace hincapié en la interrelación y la influencia bidireccional entre padres e hijos además de tener en cuenta el contexto en el que se produce dicha interacción. Es importante recalcar que no se presentan Estilos Educativos Parentales fijos o "puros", sino que los Estilos Educativos Parentales suelen ser mixtos y dinámicos durante el desarrollo del niño.

Uno de los modelos explicativos más destacables de los Estilos Educativos Parentales y pionero en este ámbito, es el de Baumrind (1996), quien se centró en el estudio de la autoridad en el ámbito de las relaciones padres-hijos. Su trabajo representa un punto de referencia fundamental en el campo del apoyo y control parental, la autonomía y el desarrollo del niño. De la combinación de las variables control, afecto y comunicación, Baumrind distingue tres Estilos Educativos 
Tabla 1

Características de los Estilos Educativos Parentales Siguiendo a Baumrind ( 1996)

\begin{tabular}{|c|c|c|c|}
\hline \multicolumn{3}{|c|}{ Estilo Educativo Parental } \\
\hline Características & Permisivo & Autoritario & Democrático \\
\hline $\begin{array}{c}\text { Niveles de afecto y } \\
\text { comunicación }\end{array}$ & Altos & Bajos & Altos \\
\hline Grado de control & Escaso & Alto & Ajustado \\
\hline Nivel de exigencia & Bajo & Alto & Elevado \\
\hline Número de normas y límites & Bajo & Elevado & \\
\hline
\end{tabular}

Parentales, mediante los que los progenitores controlan la conducta de sus hijos: a) Estilo permisivo, b) Estilo autoritario y c) Estilo democrático o autoritativo.

El estilo permisivo se caracteriza porque los padres muestran afecto y comunicación, pero escaso control en cuanto al establecimiento de normas y dificultades para establecer límites. Si bien proporcionan gran autonomía al hijo, son poco exigentes en cuanto a las expectativas de madurez y responsabilidad en la ejecución de las tareas. El estilo autoritario se ajustaría a aquellos casos en los que los padres muestran bajos niveles de comunicación y afecto y tratan de influir, controlar y evaluar el comportamiento y actitudes de sus hijos siguiendo patrones rígidos previamente delimitados. Presentan un alto nivel de control y de exigencias de madurez hacia sus hijos. El estilo democrático se caracteriza por la comunicación bidireccional y el afecto. Los padres tienden a dirigir la actividad del niño a través del razonamiento y la negociación de forma racional. Parten de una aceptación de los derechos y deberes como padres, así como de los derechos y deberes de los niños, lo que Baumrind consideraba como una «reciprocidad jerárquica» (cada sujeto tiene derechos y responsabilidades con respecto al otro). Es un estilo en el que se desarrolla la autonomía e independencia y se favorece la responsabilidad social.

Posteriormente, otros autores han aportado nuevas dimensiones o clasificaciones. MacCoby y Martin (1983) teniendo en cuenta las dimensiones de control o exigencia y grado de afecto o sensibilidad de los padres, distinguen cuatro Estilos Educativos Parentales: autoritario-recíproco, autoritario-represivo, permisivo-indulgente y permisivo-negligente. Concretamente, el estilo permisivo-negligente se ha asociado en algunos trabajos a un tipo de maltrato y ha sido analizado en diversos estudios e investigaciones (p.e., López-Soler et al., 2009; Oliva et al., 2008).

Hacia fines de los años 90, surge un nuevo enfoque en el modo de entender las interacciones familiares desde una perspectiva bidireccional, denominado Modelo de Construcción Conjunta o de Influencias Múltiples de Palacios (1999). Este modelo defiende que las relaciones entre padres e hijos son bidireccionales y que las prácticas educativas sólo son eficaces si se adecúan a la edad de los hijos y promocionan su desarrollo (Franco et al., 2014). Todos los estudios relacionados con el modelo de Palacios tienen 
una base común que es la vinculación entre Estilos Educativos Parentales y el desarrollo social, académico, conductual, emocional y moral de los hijos.

Para recoger el concepto de bidireccionalidad en las relaciones familiares, Oliva et al. (2008), deciden utilizar el nombre de Estilos Relacionales. La utilización del término Estilo Relacional en lugar del término clásico, tiene la finalidad de resaltar ese carácter interactivo, dando importancia al tipo de interacción, y no únicamente a una serie de comportamientos o actitudes parentales. Estos autores también tienen en cuenta que los hijos no son sujetos pasivos, sino agentes activos en el proceso de socialización familiar (Oliva et al., 2008).

En cualquier caso, e independientemente del modelo seguido, cabe destacar que tal y como ya apuntó Baumrind (1996) y otros autores posteriormente (p.e., Andrade-Palos et al., 2012; Belsky et al., 2005), el Estilo Educativo Parental guarda relación con el desarrollo de cualidades y características en el niño. Aparentemente, los padres que siguen un estilo permisivo forman niños alegres y vitales, aunque con altos niveles de conducta antisocial y con bajos niveles de madurez y éxito personal (Andrade-Palos et al., 2012). El estilo autoritario es el que tiene repercusiones más negativas sobre la socialización de los hijos, como la falta de autonomía personal y creatividad, menor competencia social o baja autoestima (Belsky et al., 2005). Además, este estilo autoritario es considerado como un factor de riesgo para el padecimiento en los hijos de síntomas depresivos y problemas como agresividad, hiperactividad o delincuencia (Andrade-Palos et al., 2012). Por último, las características del estilo democrático están relacionadas con un mejor ajuste psicosocial del menor. En este sentido, este Estilo Educativo Parental correlaciona positivamente con un estado emocional estable y alegre, una elevada autoestima y autocontrol y menor nivel de estrés (García-Linares et al., 2011). No obstante, algunos trabajos sugieren que el estilo más idóneo y que mejor favorece el ajuste emocional de los niños, sería el permisivo-indulgente frente al democrático (García y Gracia, 2010).

En este contexto, gana relevancia el estudio de cómo los Estilos Educativos Parentales se relaciona con la aparición de dificultades o incluso trastornos en la infancia y la adolescencia.

Estudios recientes como el de PérezFuentes et al. (2019), respaldan la influencia de los Estilos Educativos Parentales en el ajuste psicosocial de los hijos. En este estudio Pérez-Fuentes et al. (2019) afirman que el Estilo Educativo Parental óptimo presenta variaciones dependientes del entorno cultural. Estos autores concluyen que un estilo basado en la alta calidez y el bajo rigor (como el estilo indulgente), es el que mayor correlación guarda con un mejor ajuste psicosocial de los menores junto con una mejor evolución escolar, cognitiva, conductual y motivacional. También, García et al. (2020) cuestionan si el rigor parental contribuye o no al ajuste psicosocial de los hijos en todos los contextos culturales. En este caso, García et al. (2020) observan que el estilo indulgente se relaciona con un autoconcepto más favorable y mayor bienestar.

Teniendo en cuenta lo anterior, el objetivo de este estudio fue realizar una revisión en torno a la temática de los Estilos Educativos Parentales y su relación con trastornos en la infancia y adolescencia.

\section{MÉTODO}

\section{PROCEDIMIENTO}

Se sigue una secuencia metodológica ordenada, depurando fuentes de información no confiables. Para realizar el proceso de búsqueda de información y selección, se utilizaron buscadores especializados, repositorios y bases de datos como la Web of Science, Google Académico, Psyclnfo, Pubmed. 
La sintaxis de búsqueda fue: "educational styles" AND "disorders" AND "children" AND "adolescents". De los más de 500 documentos generados de la búsqueda con los términos anteriores, se seleccionaron aquellos que cumplían como criterios de inclusión: (1) Trabajos empíricos, revisiones bibliográficas y metaanálisis; (2) Publicados en inglés o en castellano; (3) La muestra de estudio corresponde al periodo de los 3 a los 18 años; (4) Estudios publicados desde 2011 hasta 2021.

Los criterios de exclusión de documentos han sido los siguientes: (1) Estudios anteriores al año 2011; (2) Estudios de carácter retrospectivo o centrados en la edad adulta; (3) Estudios que no respondían a criterios científicos.

Se identificaron 73 artículos, de los que finalmente se seleccionaron 16 trabajos que cumplían con los criterios descritos y de los que se podía acceder al texto completo.

\section{MUESTRA}

De los 16 estudios seleccionados, un trabajo se centró en los Estilos Educativos Parentales y la discapacidad intelectual (Fuenzalida-Ríos et al., 2017). Cuatro trabajos estaban enfocados específicamente

\begin{tabular}{l}
$\begin{array}{c}\text { Artículos identificados con las bases de datos: Web of } \\
\text { Science, Google Académico, Psyclnfo, Pubmed } \\
(n=73)\end{array}$ \\
\hline \multicolumn{2}{|c|}{$(n=33)$} \\
\hline Artículos después de excluir duplicados \\
$(n=17)$ \\
\hline Referencias irrelevantes \\
$(n=17)$ \\
\hline Artículos incluidos en la revisión sistemática \\
$(n=16)$
\end{tabular}

en la relación entre los Estilos Educativos Parentales y el TDAH (Çolak et al., 2020; Hidalgo-Vicario y Sánchez-Santos, 2014; Rioux et al., 2019; Santurde del Arco y del Barrio del Campo, 2014). Otros cinco trabajos, profundizaron en la relación entre los Estilos Educativos Parentales y la sintomatología internalizante como la ansiedad y la depresión (Aguilar-Yamuza et al., 2019; Antón-San Martín et al., 2016; Franco et al., 2014; Rosa-Alcázar et al., 2014; Sahithya y Vijaya, 2021). En lo que respecta a los Estilos Educativos Parentales y la sintomatología externalizante como los problemas conductuales, se recogieron dos trabajos (Álvarez García et al., 2016; RuizHernández et al., 2018). Un trabajo analizó la combinación entre los Estilos Educativos Parentales, el TDAH y la ansiedad (Meyer et al., 2021). Finalmente, tres estudios se enfocaron en la relación entre Estilos Educativos Parentales y aspectos como el ajuste psicosocial y el bienestar (GuijarroGallego et al., 2021; Mónaco et al., 2019; Pérez-Fuentes et al., 2019).

\section{RESULTADOS}

Hay diversos elementos que se relacionan con la aparición de trastornos específicos en la niñez y adolescencia, pero uno de los que muestra mayor repercusión en la conducta de los hijos es el Estilo Educativo Parental (Kendall et al., 2006). En este sentido, siguiendo a Fuentes et al. (2015) los Estilos Educativos Parentales que ponen en marcha estrategias basadas en el afecto y la implicación, promueven un adecuado ajuste social en los niños y adolescentes.

De la revisión realizada sobre la relación de los Estilos Educativos Parentales y los trastornos en la infancia y la adolescencia, destacan trabajos como el de FuenzalidaRíos et al. (2017) quienes analizaron los niveles de permisividad y sobreprotección en jóvenes con discapacidad intelectual de Chile y España. Sus resultados reflejaron diferencias culturales y un mayor nivel de permisividad en aquellas familias españolas 
con hijos con discapacidad intelectual.

En relación con trastornos del desarrollo, como el trastorno por déficit de atención con hiperactividad (TDAH), con un alto índice de prevalencia entre la población infanto-juvenil, estudios como el de Santurde del Arco y del Barrio del Campo (2014) han destacado la implicación de los Estilos Educativos Parentales en los niños y adolescentes con TDAH. Estos autores destacan que es pertinente favorecer la asunción de prácticas de crianza que garanticen la eficacia parental y que beneficien una evolución positiva de la sintomatología del TDAH. También, Rioux et al. (2019) subrayaron que las prácticas de crianza positivas predicen niveles más bajos de hiperactividad-impulsividad y mejor nivel atencional a los siete años. En esta línea, Hidalgo-Vicario y Sánchez-Santos (2014) recogen algunos factores familiares de riesgo y protección que pueden favorecer o dificultar la afectación del TDAH en el contexto familiar. Como factores de riesgo destacaron por ejemplo los vínculos familiares débiles, falta de disciplina y límites claros, ambiente familiar conflictivo, psicopatología en los padres, o baja autoestima de los progenitores. Por otro lado, como factores de protección indicaron la facilidad para la comunicación familiar, la autoestima adecuada de los progenitores, el buen ambiente familiar y presencia de límites, el control interno bien establecido, o la ausencia de psicopatología familiar.

También en relación con el TDAH, Çolak et al. (2020) realizaron un estudio cuyo objetivo fue analizar la relación entre el nivel de severidad de los síntomas del TDAH y las actitudes de los padres de niños diagnosticados con TDAH. Sus resultados reflejaron que las actitudes paternas juegan un papel clave en relación a la gravedad de la sintomatología del TDAH, así como en el pronóstico del trastorno.

Otros estudios han observado la influencia del Estilo Educativo Parental en otros trastornos diferentes del TDAH. Por ejemplo, Franco et al. (2014) estudiaron la relación entre los Estilos Educativos Parentales, la ansiedad y conductas disruptivas en niños. Sus resultados mostraron que determinadas actitudes y pautas de crianza parental, influyen de manera significativa en el desarrollo y mantenimiento de conductas disruptivas y alteraciones emocionales en los hijos. Antón-San Martín et al. (2016), realizaron una investigación con el objetivo de analizar la interacción entre los Estilos Educativos Parentales percibidos por los hijos, la intensidad de la psicopatología presentada y las dimensiones sintomáticas internalizante-externalizante en una muestra clínica de adolescentes de entre 13 y 18 años. Los resultados indicaron que únicamente dos dimensiones del Estilo Educativo Parental percibido estaban relacionadas con la intensidad psicopatológica: 1) El humor, de forma positiva; y 2) El control psicológico, de forma negativa (vinculado con sintomatología externalizante).

Aguilar-Yamuza et al. (2019) profundizaron en la relación existente entre determinadas variables familiares, el estilo Educativo Parental y la depresión y ansiedad en los hijos. Los resultados indicaron que determinadas actitudes y pautas de crianza (satisfacción en la crianza, disciplina de ambos progenitores,...) y variables familiares (implicación de ambos progenitores, orden del niño entre sus hermanos,...), pueden influir de manera significativa en la presencia y desarrollo de algunos trastornos internalizantes en los hijos. En esta línea, Rosa-Alcázar et al. (2014), observaron que los Estilos Educativos Parentales se relacionan con la aparición de trastornos internalizantes durante la infancia, como la ansiedad y la depresión. Sahithya y Vijaya (2021) destacan que la permisividad parental es un factor de riesgo para el desarrollo de problemas de ansiedad en los niños, mientras que la autoridad materna y la sociabilidad del menor son elementos protectores contra este tipo de trastornos.

Con respecto a otros trastornos de carácter externalizante, siguiendo a Álvarez García et al. (2016), las prácticas educativas y relaciones ejercidas por los padres pueden resultar un factor predictor del comportamiento antisocial 
en la adolescencia. Al contrario, los Estilos Educativos Parentales caracterizados por el afecto, la comunicación y el apoyo, se asocian negativamente con el comportamiento antisocial en los niños, incluido el uso de drogas, el comportamiento delictivo o el acoso escolar (Ruiz-Hernández et al., 2018).

Meyer et al. (2021) tuvieron en cuenta tanto el TDAH como la ansiedad y su relación con los estudios educativos parentales. Estos autores destacaron que la crianza sobreprotectora media la relación entre el TDAH en la primera infancia y los síntomas de ansiedad, y tanto el TDAH como la ansiedad en este período se relacionan con Estilos Educativos Parentales en los que prima la sobreprotección. En este estudio se constata que los primeros síntomas del TDAH serían predictores de una paternidad sobreprotectora, lo cual, correlaciona positivamente con síntomas de ansiedad en la infancia y adolescencia

A nivel general, en términos de equilibrio psicosocial, estabilidademocional, satisfacción vital o bienestar, se recogieron trabajos como el de Guijarro-Gallego et al. (2021), PérezFuentes et al. (2019) y Mónaco et al. (2019). Guijarro-Gallego et al. (2021), realizaron un estudio en cuanto a la relación existente entre los Estilos Educativos Parentales y el equilibrio psicosocial de los menores. Pérez-Fuentes et al. (2019) analizaron la relación entre el Estilo Educativo y la satisfacción vital en adolescentes, y concluyeron que la forma de percibir el estado de ánimo y el afecto de los padres por parte de los hijos son dos aspectos que presentan una correlación sólida con la satisfacción vital, mientras que el control psicológico correlaciona negativamente con la misma. En cuanto a la satisfacción vital y el Estilo Educativo de los padres, Pérez-Fuentes et al. (2019) indicaron que la satisfacción aumenta junto con los niveles de afecto y comunicación, por el contrario, disminuye en los casos en que hay un aumento del control psicológico. Finalmente, Mónaco et al. (2019) y Pérez-Fuentes et al. (2019) destacaron que la relevancia del apoyo de los padres (afecto y comunicación), podría resultar un predictor importante de la satisfacción vital de los hijos. Por lo tanto y como se desprende de estos últimos estudios, el Estilo Educativo Parental basado en la promoción de relaciones cálidas y la capacidad de afrontar mediante el razonamiento las dificultades que van surgiendo en el desarrollo de los menores son elementos fundamentales para un desarrollo psicosocial equilibrado (Mónaco et al., 2019; Pérez-Fuentes et al. (2019).

\section{DISCUSIÓN}

El presente trabajo planteó realizar una revisión en torno a los Estilos Educativos Parentales y su relación con trastornos en la infancia y adolescencia. Los estudios revisados subrayan el papel fundamental de la influencia paterna y materna sobre los hijos, tomando gran relevancia las prácticas educativas y los diferentes Estilos Educativos Parentales que las enmarcan (p.e., Fuentes et al., 2015; Sahithya y Vijaya, 2021).

Los Estilos Educativos Parentales se configuran como un pilar básico que resulta determinante en el desarrollo psicosocial de los hijos, pudiendo incluso jugar un papel clave en la aparición de trastornos en los menores tanto en la infancia como en la adolescencia (Lemos-Giráldez, 2003). Algunos de estos trastornos pueden ser la ansiedad, la depresión, las alteraciones emocionales, o el TDAH (Aguilar-Yamuza et al., 2019; Colak et al., 2020; Meyer et al., 2021). En este sentido, los Estilos Educativos Parentales son considerados por muchos autores como una variable moduladora que protege o pone en riesgo al niño ante la posible aparición de alteraciones (Hidalgo-Vicario y SánchezSantos, 2014; Kendall et al., 2006).

Se puede concluir a modo de síntesis que, cuando los padres utilizan Estilos Educativos Parentales caracterizados por el afecto y la implicación, se promueve un adecuado ajuste psicosocial en los menores; mientras que las pautas relacionadas con menor grado de afecto, se situarían en el polo opuesto, 
correlacionando positivamente en muchas ocasiones con problemas internalizantes y externalizantes (Aguilar-Yamuza, 2019). Concretamente, en el caso del TDAH, cada vez más presente en la población infantil y juvenil, los estudios consultados indican que las prácticas positivas de crianza favorecen la evolución positiva y el pronóstico del trastorno (Franco et al., 2014).

La influencia de los Estilos Educativos Parentales también ha quedado de manifiesto su implicación en las conductas antisociales en la adolescencia (Álvarez-García et al., $2016)$. En este sentido, las prácticas educativas ejercidas por los progenitores pueden resultar un factor predictor del comportamiento antisocial en esta etapa evolutiva. Por el contrario, unas prácticas parentales basadas en el afecto, comunicación y apoyo, actuarían como elementos protectores respecto al comportamiento antisocial en adolescentes (incluyendo el uso o abuso de drogas, delincuencia o acoso escolar).

No debemos olvidar algunas limitaciones del presente estudio como la necesidad de incluir descriptores más específicos relacionados con la temática de estudio, la ausencia de análisis estadísticos de metaanálisis que permitiesen comparar los datos de las investigaciones, o la no inclusión de estudios no publicados en revistas científicas, disertaciones o tesis de grado y postgrado en la temática de interés.

Finalmente, a pesar de las limitaciones, los resultados de este trabajo contribuyen a poner de manifiesto el interés o relevancia del Estilo Educativo Parental en el desarrollo del niño. En este sentido, una de las implicaciones principales del presente trabajo se sitúa en la posibilidad de establecer recomendaciones específicas a las familias de niños con diferentes perfiles (como el TDAH, la ansiedad, o los problemas de comportamiento).

\section{- Conflicto de intereses}

Los autores declaran no tener ningún conflicto de interés.

\section{REFERENCIAS}

*Aguilar-Yamuza, B., Raya-Trenas, A. F., Pino-Osuna, M. J., \& Herruzo-Cabrera, J. (2019). Relación entre el estilo de crianza parental y la depresión y ansiedad en niños entre 3 y 13 años. Revista de Psicología Clínica con Niños y Adolescentes, 6(1), 36-43. https://doi.org/10.21134/ rpcna.2019.06.1.5

*Álvarez-García, D., Barreiro-Collazo, A., Dobarro, A., García, T., \& Antúnez, A. (2016). Parenting style dimensions as predictors of adolescent antisocial behavior. Frontiers in Psychology, 13(7), 13-83. https://doi.org/10.3389/ fpsyg.2016.01383

Andrade-Palos, P. A., Betancourt-Ocampo, D. B., Vallejo-Casarín, A., Celis-Ochoa, B. S., \& Rojas-Rivera, R. M. (2012). Prácticas parentales y sintomatología depresiva en adolescentes. Salud Mental, 35(1), 29. 36.

*Antón-San Martín, J. M., Seguí-Durán, D., Antón-Torre, L., \& Barrera-Palacios, A. (2016). Relación entre estilos parentales, intensidad psicopatológica y tipo de sintomatología en una muestra clínica adolescente. Anales de Psicología 32(2), 417-423. https://dx.doi.org/10.6018/ analesps.32.2.203871

Aroca-Montolío, C., \& Cánovas-Leonhardt, P. C. (2012). Los Estilos educativos parentales desde los modelos interactivo y de construcción conjunta: Revisión de las investigaciones. Teoría de la Educación, 24(2).

Baumrind, D. (1996). The discipline controvery revised. Family Relations, 45(4), 405-414. https://doi.org/10.2307/585170.

Bersabé, R., Fuentes, M., \& Motrico, E. (2001). Análisis psicométrico de dos escalas para evaluar estilos educativos parentales. Psicothema, 13(4), 678-684.

Belsky, J., Sligo, J., Jaffee, S. R., Woodward, L. \& Silva, P. A. (2005). Intergenerational transmission of warm-sensitivestimulating parenting: A prospective study of mothers and fathers of 3 yearsolds. Child Development, 76(2), 384396. https://doi.org/10.1111/i.1467. 8624.2005.00852.x 
*Rioux, C., Murray, J., Castellanos-Ryan, N., Séguin, J. R., Tremblay, R. E., \& Parent, S. (2019). Moderation of parenting by inhibitory control in the prediction of the common and unique variance of hyperactivity-impulsivity and inattention. Development and Psychopathology, 32(3), 909-921. https://doi.org/10.1017 $\angle$ S0954579419000774

Cava, M. J., \& Musitu, G. (2001). La familia y la educación. Octaedro.

*Çolak, M., Şireli-Bingöl, Ö., Esenkaya,Usta, Z., \& Kiliç, B. G. (2020). Association of attention deficit hyperactivity disorder symptoms of parents with parental attitudes. Revista de investigación académica en medicina 10(3), 258-263. https//:doi. org/10.4274/iarem.galenos.2020.3505

Fontana-Abad, M., Gil-Cantero, F., \& Reyero, D. (2013). La perspectiva pedagógica de la vida familiar. Un enfoque normativo. Estudios sobre Educación, 25, 115-132.

*Franco, N., Pérez-Nieto, M., \& de Dios, M. J. (2014). Relación entre los estilos de crianza parental y el desarrollo de ansiedad y conductas disruptivas en niños de 3 a 6 años. Revista de Psicología Clínica con Niños y Adolescentes, $1(2)$, 149-156.

Fuentes, M. C., García, F., Gracia, E., \& Alarcón, A. (2015). Los estilos parentales de socialización y el ajuste psicológico. Un estudio con adolescentes españoles. Revista de Psicodidáctica, 20(1), 117-138. https://doi.org/10.1387/ RevPsicodidact. 10876

*Fuenzalida-Ríos, I., Penelo, E., \& BrunGasca, C. (2017). Estilos educativos y psicopatología en niños y adolescentes con discapacidad intelectual de Chile y España. Quaderns de Psicología, 19(1), 101-112. https://doi.org/10.5565/rev/ gpsicologia. 1389

García, F., \& Gracia, E. (2010). ¿¿Qué estilo de socialización parental es el idóneo en España? Un estudio con niños y adolescentes de 10 a 14 años. Infancia y Aprendizaje, 33(3), 365-384. https://doi/ org: $10.1174 / 021037010792215118$

García, O. F., Fuentes, M. C., Gracia, E., Serra, E., \& Garcia, F. (2020). Parenting warmth and strictness across three generations: Parentig styles and psychosocial adjustment. International Journal of environmental research and public health. 17(20), e7487. https://doi. org/10.3390 / ijerph 17207487

García-Linares, M. C., Cerezo Rusillo, M. T., de la Torre Cruz, M. J., Carpio Fernández, M. V., \& Casanova Arias, P. F. (2011). Prácticas educativas paternas y problemas internalizantes en adolescentes españoles. Psicothema, 23(4), 654-659.

Grusec, J. E., \& Hastings, P. D. (2007). Handbook of socialization: Theory and research. Guilford Press.

*Guijarro-Gallego, A., Martínez Pérez, A., Fernández Fernández, V., AlcántaraLópez, M., \& Castro Sáez, M. (2021). Life satisfaction in adolescents: Relationship with parental style, peer attachment and emotional intelligence. Electronic Journal of Research in Educational Psychology, 19(1), 51 -74. https://doi.org/10.25115/ ejrep.v19i53.3425

*Hidalgo-Vicario, M. I., \& Sánchez-Santos, L. (2014). Trastorno por déficit de atención e hiperactividad. Manifestaciones clínicas y evolución. Diagnóstico desde la evidencia científica. Pediatría Integral, 18(9), 609623. https://doi.org/10.5944/rppc. vol.14.num.2.2009.4067

Izzedin-Bouquet, R., \& Pachajoa-Londoño, A., (2009). Pautas, prácticas y creencias acerca de crianza... ayer y hoy. Liberabit Revista de Psicología, 15(2), 109-115.

Jorge, E., \& González, M. C. (2017). Estilos de crianza parental: una revisión teórica. Informes Psicológicos, 17(2), 39-66.

Kendall, P. C., Hedtke, K. A., \& Aschenbrand, S. G. (2006). Anxiety disorders. En D. A. Wolfe, \& E. J. Mash (Eds.), Behavioral and emotional disorders in adolescents. Nature, assessment, and treatment, (25929). Guilford Press.

Lemos-Giráldez, S., (2003). La psicopatología de la infancia y la adolescencia: consideraciones básicas para su estudio. Papeles del Psicólogo, 24(85), 19-28.

López-Soler, C., Puerto, J. C., López-Pina, J. A., \& Prieto, M. (2009). Percepción de los estilos educativos parentales e inadaptación en menores pediátricos. Anales de Psicología, 25(1), 70-77. 
Maccoby, E. E., \& Martín, J. A. (1983). Socialization in the context of the family: Parent-child interaction. En E. M. Hetherington \& P.H. Mussen (Eds), Handbook of child psychology: Socialization, personality and social development (pp. 1-101). Wiley.

*Meyer, A., Kegley, M., \& Klein, D.N. (2021). Overprotective parenting mediates the relationship between early childhood ADHD and anxiety symptoms: Evidence from a cross-sectional and longitudinal study. Journal of Attention Disorders, 26(2), 319-327. doi: https://doi. org/10.1177/1087054720978552

*Mónaco, E., Schoeps, K., \& MontoyaCastilla, I. (2019). Attachment styles and well-being in adolescents: How does emotional development affect this relationship? International Journal of Environmental Research and Public Health, 16(14), e2554. https://doi.org/10.3390/ ijerph 16142554

Oliva, A., Parra, A., \& Arranz, E. (2008). Estilos relacionales parentales y ajuste adolescente. Infancia y Aprendizaje, 31 (1), 93-106. https://doi. org/10.1 174/021037008783487093

Asamblea General de las Naciones Unidas. (1948). Declaración Universal de los Derechos Humanos. Publicaciones de las Naciones Unidas.

Palacios, J. (1999). La familia y su papel en el desarrollo afectivo y social. En F. López, I. Etxebarría, M. J. Fuentes, \& M. J. Ortiz (Eds.) Desarrollo afectivo y social (pp. 267-284). Pirámide.

*Pérez-Fuentes, M.C., Molero, M. M., Gázquez, J. J., Oropesa, N. F., Simón, M. M., \& Saracostti, M. (2019). Parenting pratices, life satisfaction and the role of self-esteem in adolescents. International Journal of Enviornmental research and public health, 16(20), e4045. https://doi. org/10.3390/ijerph16204045
Rodrigo, M. J., \& Palacios-González, J. (2014). Familia y desarrollo humano. Alianza Editorial.

*Rosa-Alcázar, A. I., Parada-Navas, J. L., \& Rosa-Alcázar, A. (2014). Síntomas psicopatológicos en adolescentes españoles: relación con los estilos parentales percibidos y la autoestima. Anales de Psicología, 30(1), 133142 . https://dx.doi.org/10.6018/ analesps.30.1.165371

*Ruiz-Hernández, J. A., Mora, E., LlorEsteban, B., \& Jiménez, J. A. (2018). La influencia de los estilos parentales y otras variables psicosociales en el desarrollo de los comportamientos externalizantes en adolescentes: revisión sistemática. The European Journal of Psychology Applied to Legal context, 17 (1), 9-21. https://doiorg/10.5093/ejpalc2018a 1

*Sahithya, B. R., \& Vijava Raman (2021). Parenting style, parental personality, and child temperament in children with anxiety disorders. A clinical study from India. Indian Journal of Psychological Medicine, 43(5), 382-391. https://doi. org/10.1177/0253717620973376

* Santurde del Arco, E., \& del Barrio del Campo, J. A. (2014). Papel relevante de los estilos educativos en la evolución de los síntomas del TDAH. Revista de Psicología, 1(1), 251-258. https://doi. org/10.17060/iiodaep.2014.n1.v1.368

Torío-López, S., Peña, J. V., \& Rodríguez, C. (2008). Estudios educativos parentales. Revisión bibliográfica y reformulación teórica. Teoría de la Educación. Revista Interuniversitaria, 20, 151-178. 